\title{
xviii LABORATORY METHODS OF ORGANIC CHEMISTRY
}

\section{ABBREVIATIONS}

The abbreviations of the titles of journals are those employed in British Chemical Abstracts. The abbreviated title is followed by the year, volume number (in heavy type), and page.

Annalen $\quad=$ Justus Liebig's Annalen der Chemie.

Ann. Chim. = Annales de Chimic [et de Physique].

Ber. $\quad=$ Berichte der Deutschen Chemischen Gesellschaft.

Bull. Soc. chim. = Bulletin de la Société chimique de France.

Chem. Zentr. $\quad=$ Chemisches Zentralblatt.

Helv. Chim. Acta = Helvetica Chimica Acta.

J. Amer. Chem. Soc. = Journal of the American Chemical Society.

J.C.S. $\quad=$ Journal of the Chemical Society.

J. pr. Chem. $\quad=$ Journal für praktische Chemie.

Monatsh. $=$ Monatshefte für Chemie und verwandto Teile anderer Wissenschaften.

Rec. trav. chim. $\quad=$ Recueil des travaux chimiques des Pays-Bas.

Z. angew. Chem. =Zeitschrift für angewandte Chemie.

Z. physiol. Chem. = Hoppe Seyler's Zeitschrift für physiologische Chemic. 